\title{
Experiencia de la Unidad de Medicina Reproductiva Clínica Monteblanco con el uso de corifollitropin alfa en ciclos de fecundación in vitro
}

\author{
Juan E. Schwarze M., MSCE ${ }^{1,2}$, Sonia Villa V. ${ }^{1}$, Carolina Ortega $H^{1}{ }^{1}$, Ricardo Pommer . $^{1}$ \\ ${ }^{1}$ Unidad Medicina Reproductiva Monteblanco. ${ }^{2}$ Departamento Clínico de Obstetricia y Ginecología, Universidad de \\ Santiago. Chile.
}

\section{RESUMEN}

Antecedentes: La hiperestimulación ovárica controlada (HEOC) es uno de los componentes fundamentales de los ciclos de fecundación in-vitro (FIV). HEOC se ha realizado con gonadotrofinas exógenas de uso diario, agregando un componente de estrés adicional al tratamiento. La aparición de una FSH recombinante de depósito (corifollitropin alfa) permite disminuir el estrés asociado. Los estudios publicados no han mostrados diferencias en el número de ovocitos recuperados ni en las tasas de embarazo clínico. Los estudios existentes han sido financiados en su mayoría por la industria farmacéutica. Objetivo: Presentar la experiencia de la Unidad de Medicina Reproductiva Monteblanco con el uso de corifollitropin alfa en ciclos de FIV autólogos en pacientes no seleccionadas. Método: Se analizaron el número de ovocitos recuperados y la proporción de ovocitos en metafase 2 en pacientes sometidas a HEOC con corifollitropin alfa, FSH recombinante diaria ( $\mathrm{rFSH}$ ), y la combinación de FSH recombinante y urinaria. Resultados: Se analizaron 727 ciclos de FIV: 270 con corifollitropin alfa, 33 con rFSH y 333 con combinación de FSH. No hubo diferencias significativas en la recuperación de ovocitos ni en la proporción de ovocitos en metafase 2. Al corregir por edad de la mujer y tipo de esquema de HEOC, encontramos que la edad de la mujer se asoció negativamente con el número de ovocitos recuperados, no así el tipo de esquema de HEOC. Conclusión: El uso de corifollitropin alfa en ciclos de FIV autólogos, no se diferencia significativamente de los otros esquemas de HEOC en la media de ovocitos recuperados ni en la media de ovocitos en metafase 2 obtenidos.

\section{PALABRAS CLAVE: FIV, hiperestimulación ovárica controlada, infertilidad}

\section{SUMMARY}

Background: Controlled ovarian hyperstimulation $(\mathrm{COH})$ is a main component of in-vitro fertilization (IVF) cycles. $\mathrm{COH}$ have been performed with daily exogenous gonadotropins administered, adding to treatment an additional component of stress. The appearance of a depot recombinant FSH (corifollitropin alfa) helps to reduce stress in patients undergoing IVF. No studies have shown differences in the number of retrieved oocytes or clinical pregnancy rates; however these studies have been funded by the pharmaceutical industry. Aims: To show the experience of Reproductive Medicine Unit Monteblanco with the use of corifollitropin alpha in autologous IVF cycles, in unselected patients. Methods: Our main outcome was the mean number of oocytes retrieved, and the proportion of oocytes in metaphase 2 in patients undergoing $\mathrm{COH}$ with corifollitropin alpha, daily recombinant $\mathrm{FSH}(\mathrm{rFSH})$, and a combination of $\mathrm{rFSH}$ and urinary gonadotropin. Results: We analyzed 727 IVF cycles: 270 cycles with corifollitropin alpha, 33 exclusive rFSH and 333 cycles with rFSH combination and urinary gonadotropins. We did not found any statistically significant difference in the 
mean number of oocytes recovered nor the proportion of metaphase two oocytes obtained. After adjusting for age, we did not find that the $\mathrm{COH}$ protocol influenced the mean number of oocytes recovered. Conclusion: We concluded that the use of corifollitropin alpha in autologous IVF cycles does not alter the mean number of oocytes recovered, nor the proportion of oocytes in metaphase 2.

\section{KEY WORDS: IVF, controlled ovarian hyperstimulation, infertility}

\section{INTRODUCCIÓN}

El primer niño nacido de fecundación in vitro en Chile fue reportado por el equipo del Hospital Militar liderado por el Dr. Alberto Costoya en una carta a esta misma revista en 1984 (1). Desde entonces, la cantidad de ciclos de reproducción asistida ha aumentado en forma sostenida, reportándose en el año 2011 un total de 1.918 ciclos de técnica de reproducción asistida, correspondiendo un 1.427 a ciclos iniciados de fecundación in vitro con óvulos propios.

Una de los componentes fundamentales de los ciclos de fecundación in vitro es la hiperestimulación ovárica controlada, mediante la cual se rescatan ovocitos de la atresia, y se consigue un desarrollo polifolicular. Al aumentar la cantidad de ovocitos desarrollados y recuperados, aumenta las probabilidades que al menos uno de estos ovocitos sea normal, con lo que aumenta la probabilidad de conseguir un embrión sano y, tras la transferencia de éste, un embarazo viable y un niño sano.

La hiperestimulación ovárica controlada se consigue mediante la administración de gonadotrofinas exógenas, ya sean de origen urinario o recombinante. Diversos estudios han mostrado que la administración diaria de estos fármacos y el temor a equivocarse en su administración es una fuente importante de estrés para la pareja sometida a tratamiento de reproducción asistida, lo que incluso puede repercutir negativamente en el resultado del mismo $(2,3,4)$.

Como una forma de disminuir este estrés, se desarrolló una FSH recombinante de depósito que evitaba por siete días la administración diaria de gonadotrofinas. Este nuevo fármaco, corifollitropin alfa, está compuesto por una unidad alfa que es idéntica a la de la FSH, y una subunidad beta, producto de la fusión de subunidad beta de la hormona gonadotrofina coriónica con la subunidad beta de FSH. La subunidad beta de la corifollitropin alfa tiene una vida media más larga que la FSH, requiriendo así de una menor frecuencia de administración (5).

Los estudios que compararon el número de ovocitos y proporción de ovocitos en metafase 2 , demostró que la administración de corifollitropin alfa no era inferior a la administración diaria de FSH recombinante. Asimismo, encontraron altas tasas de embarazo clínico al administrar este nuevo fármaco en un ciclo de fecundación in vitro (6).

La principal crítica que se hace a los estudios clínicos aleatorizados realizados bajo el auspicio de la industria farmacéutica es que tienen poca validez externa, ya que los participantes en los estudios son estrictamente seleccionados, de modo que tienen poca relación con los pacientes que normalmente vemos en la práctica clínica habitual $(7,8)$. Debido a lo anterior es que el objetivo de este estudio es presentar nuestra experiencia con el uso de corifollitropin alfa en ciclos de fecundación in vitro autólogos en pacientes no seleccionadas.

\section{PACIENTES Y MÉTODOS}

La Unidad de Medicina Reproductiva Monteblanco es parte de la Red Latinoamericana de Reproducción Asistida, y como tal, reporta anualmente todos sus casos al Registro Latinoamericano de Técnicas de Reproducción Asistida (RLA). En forma periódica, la unidad es visitada por dos profesionales externos, quienes revisan y certifican los procesos de las distintas técnicas de reproducción asistida, y la veracidad y consistencia de la información enviada al RLA.

La información registrada incluye datos demográficos de la paciente, características de los ciclos de reproducción asistida, resultados y complicaciones de los mismos. Para esta revisión analizamos el número de ovocitos recuperados, la proporción de ovocitos en metafase 2 y la frecuencia de fecundación de estos ovocitos en pacientes -con reserva ovárica adecuada- a las que se les administró corifollitropin alfa.

Hiperstimulación ovárica controlada. El protocolo de hiperestimulación ovárica fue como sigue. En el segundo o tercer día del ciclo menstrual, luego de descartar mediante ultrasonido que la paciente presentaba alguna lesión ovárica o endometrial que contraindicara la hiperestimulación ovárica, una matrona coordinadora administraba 150 ug de corifolitropin alfa (Elonva, MSD) sc. Siete días después, se controló el desarrollo folicular mediante ultrasonido transvaginal, y continuó la estimulación ya sea mediante la administración diaria de 200 IU de FSH recombinante (Puregon $®$, MSD). Cuando el folículo de mayor diámetro llegó a 14 $\mathrm{mm}$, se agregó la administración diaria de un an- 
tagonista de $\mathrm{GnRH}$, ganirelix (Orgalutran®, MSD), para evitar la luteinización prematura. Una vez que al menos dos folículos llegaron a un diámetro promedio de $17 \mathrm{~mm}$, se indujo la ovulación mediante la administración de $0,5 \mathrm{mg}$ de acetato de leuprolida (Lupron, Abbott).

Los otros esquemas consistieron en el uso de 200 IU sc de FSH recombinante (Puregon $®$, MSD; Gonal-F®, Merck-Serono) en forma diaria, hasta determinar el uso de antagonista e inducción de maduración ovárica final. El tercer esquema utilizado fue el uso asociado de 200 IU Sc de FSH recombinante (Puregon $\AA$, MSD; Gonal-F®, MerckSerono) con 75 IU de HMG (Menopur®, Ferring), hasta alcanzar los mismos criterios de asociación de antagonsita $\mathrm{GnRH}$ e inducción de maduración ovocitaria final.

Procedimiento de laboratorio de embriología. Entre 35 a 37 horas de inducido la maduración final de los ovocitos, éstos fueron recuperados mediante aspiración folicular guiada por ultrasonido bajo anestesia. Los ovocitos fueron identificados mediante un microscopio estereoscópico y luego de su denudación se catalogados mediante la identificación del primer polocito, como maduros (en metafase II) o inmaduros (metafase I o vesícula germinativa). Luego, después de dejarlos estabilizar seis horas en medio de cultivo, fueron inyectados con un espermatozoide con la ayuda de un microscopio invertido (modelo y marca) y micromanipuladores (Narishige, Japón). Diez y ocho horas luego de la inyección se comprobó la presencia de dos pronúcleos, señal inequívoca de fecundación exitosa. En ningún caso se transfirieron embriones, los que fueron vitrificados para transferir en un subsecuente ciclo cuando correspondiera.

Análisis estadístico. Se presenta la información como promedios y desviación estándar. Para comparar promedios, confirmado que la distribución no es normal, se utilizaron test estadísticos no parametricos (Kruskal-Wallis). Realizamos regresión lineal considerando como variable dependiente el número de ovocitos recuperados en metafase II, y como variables independientes la edad de la mujer y el esquema de hiperestimulación ovárica. Los esquemas de hiperestimulación ovárica analizados fueron: a) Elonva, b) FSH recombinante diaria, c) combinación de $\mathrm{FSH}$ recombinante y urinaria.

\section{RESULTADOS}

Analizamos 727 ciclos realizados entre el $1^{\circ}$ de enero del 2014 y 31 de junio del 2015. De ellos, 270 correspondieron a ciclos con Elonva $\circledR$, 33 a ciclos con el uso exclusivo de rFSH, y 333 a ciclos en los que usó la combinación de FSH recombinante y urinaria.

En la Tabla I se muestran los resultados de los ciclos. La diferencia en la media de ovocitos recuperados y de ovocitos en metafase 2 no alcanza significancia estadística ( $p=0,267$ y $p=0,592$, respectivamente).

Realizamos una regresión lineal para corregir por la edad de la mujer, ocupando como referencia el uso combinado de FSH recombinante y urinaria. La edad de la mujer se asoció negativamente con el número de ovocitos recuperados $(p<0,001)$, mientras que el uso Elonva $(p=0,839)$ y de $F S H$ recombinante exclusivo $(p=0,777)$ no se asoció en forma significativa a la media de ovocitos en metafase 2 . No se registraron casos de hiperestimulación ovárica en ninguno de los grupos.

\section{DISCUSIÓN}

Presentamos nuestra experiencia con el uso de corifollitropin alfa en 270 pacientes no seleccionadas, sometidas a hiperestimulación ovárica controlada para realizar un ciclo de fecundación in vitro autólogo. Encontramos que comparados con otros esquemas de hiperestimulación ovárica controlada, el uso de corifollitropin alfa no se asoció a un cambio significativo en el número de ovocitos recuperados ni el número de ovocitos en metafase 2.

Tabla I

RESUMEN DE LOS CASOS REALIZADOS EN LA UNIDAD DE MEDICINA REPRODUCTIVA CLÍNICA MONTEBLANCO

\begin{tabular}{lccc}
\hline Variable & Elonva & rFSH & rFSH/uFSH \\
\hline Número & 270 & 33 & 333 \\
Edad de la mujer (años) & $38 \pm 7$ & $38 \pm 5$ & $37 \pm 4$ \\
Número ovocitos recuperados & $9,9 \pm 7,0$ & $9,6 \pm 8,2$ & $10,9 \pm 8,6$ \\
Número ovocito metafase II & $8,7 \pm 6,0$ & $9,2 \pm 6,4$ & $9,0 \pm 6,2$ \\
\hline
\end{tabular}

Promedio \pm desviación estándar 
Tabla II

REGRESIÓN LINEAL PARA PREDECIR NÚMERO DE OVOCITOS RECUPERADOS EN METAFASE II

\begin{tabular}{lcc}
\hline Factor & Cuociente de regresión & Valor $p$ \\
\hline Edad & $-0,26(-0,34$ a $-0,17)$ & $<0,001$ \\
Elonva $\left(^{*}\right)$ & $-0,11(-1,16$ a 0,94$)$ & 0,839 \\
FSHr solo $\left(^{*}\right)$ & $-0,36(-2,84$ a 2,12$)$ & 0,777
\end{tabular}

$\left(^{*}\right)$ Se tomó la combinación de FSH recombinante y urinaria como referencia

La gran ventaja del presente estudio es que se trata de un análisis simple del uso regular de corifoIlitropin alfa en pacientes no seleccionadas. Todos los reportes publicados, según nuestro conocimiento, se refieren a estudios clínicos, en los que las pacientes son cuidadosamente seleccionadas entre aquellas con buen pronóstico, lo que les resta validez externa. Probablemente debido a esto, es que muchos de los especialistas en medicina reproductiva son reluctantes a ocupar nuevos fármacos o esquemas, en espera de la experiencia de otros en situaciones más parecidas a su práctica habitual.

Nuestro estudio presente varias desventajas. Probablemente la principal es que se trata de un análisis retrospectivo, en el que el uso de cada uno de los esquemas puede reflejar la preferencia de cada uno los médicos tratantes de la unidad, más que una distribución aleatoria de la misma. Sin embargo, consideramos que los resultados son consistentes con un uso no sesgado de cada uno de los esquemas.

Dado que toda la evidencia, tanto en estudios prospectivos aleatorizados como retrospectivos como el presente, demuestran que el uso de corifollitropin alfa es similar al uso de otros esquemas, queda por determinar cuál es la experiencia emocional de las mujeres sometidas a los diferentes esquemas de hiperestimulación ovárica controlada.

\section{CONCLUSIÓN}

No encontramos que el uso de corifollitropin alfa estuviera asociado a una diferencia estadísticamente significativa en la media de ovocitos recuperados ni en la media de ovocitos en metafase 2. Esperamos que esta experiencia de confianza a otros especialistas a usar esta nueva droga, dado que disminuiría así el número de inyecciones a aplicar.

\section{REFERENCIAS}

1. Costoya, A. Schmitt JM, Rey M, Dujovne S, Sánchez MI, Gadán A, et al. [Pregnancy obtained by in vitro fertilization and embryo transfer]. Rev Chil Obstet Ginecol 1984;49(3),206-16.

2. Ebbesen SM1, Zachariae R, Mehlsen MY, Thomsen D, Højgaard A, Ottosen L, Petersen T, Ingerslev HJ. Stressful life events are associated with a poor in-vitro fertilization (IVF) outcome: a prospective study. Hum Reprod 2009;24:2173-82.

3. Csemiczky G, Landgren B M, Collins A. The influence of stress and state anxiety on the outcome of IVFtreatment: psychological and endocrinological assessment of Swedish women entering IVF-treatment. Acta Obstet Gynecol Scand 2000;79;113-8.

4. Poehl M, Bichler K, Wicke V, Dörner V, Feichtinger W. Psychotherapeutic counseling and pregnancy rates in in vitro fertilization. J Assist Reprod Genet 1999;16:302-5.

5. Loutradis D, Drakakis P, Vlismas A, Antsaklis A. Corifollitropin alfa, a long-acting follicle-stimulating hormone agonist for the treatment of infertility. Curr Opin Investig Drugs 2009;10:372-80.

6. Seyhan A, Ata B. The role of corifollitropin alfa in controlled ovarian stimulation for IVF in combination with GnRH antagonist. Int J Womens Health 2011;3:24355.

7. Fahey $T$, Stocks N. Antibiotics for children with upper respiratory tract infections. JAMA 1998;280:1399400. Author reply 1998;1401-2.

8. Patsopoulos NA. A pragmatic view on pragmatic trials. Dialogues Clin Neurosci 2011;13:217-24. 